\title{
Systemic lupus erythematosus and invasive thymoma: report of two cases
}

\author{
M. M. STEVEN ${ }^{1}$ M. L. WESTEDT ${ }^{1}$ F. EULDERINK ${ }^{2}$ H. M. HAZEVOET, \\ J. H. DIJKMAN ${ }^{3}$, AND A. CATS
}

From the Departments of ${ }^{1}$ Rheumatology, ${ }^{3}$ Respiratory Diseases, and ${ }^{2}$ Pathology, Academisch Ziekenhuis Leiden, Rijnsburgerweg 10, 2333 AA Leiden, The Netherlands

SUMmARY Two cases of invasive thymoma in patients with systemic lupus erythematosus (SLE) are described. In both instances the suspected diagnosis of a mediastinal tumour proved difficult to confirm. Neither surgical removal of the thymoma in one case nor irradiation in the other had any apparent clinical or serological effect on the course of the SLE. Both patients subsequently suffered from respiratory problems and the distinction between recurrent tumour and pulmonary involvement by SLE proved difficult. One patient had a good response, however, to pulse methylprednisolone, but the other later developed recurrence of tumour and died of Pneumocystis carinii infection following cytotoxic therapy three years after discovery of the tumour.

Key words: mediastinal neoplasm, methylprednisolone, Pneumocystis carinii.

Systemic lupus erythematosus (SLE) is a multisystem disorder associated with several immunological phenomena, including the production of large amounts of autoantibodies. These phenomena have been attributed to a loss of the control over B lymphocytes which is normally exerted by $T$ lymphocytes, ${ }^{1}$ but the reason for this imbalance is unknown. Tumours of the thymus are rare but have been reported in concurrence with a variety of putative autoimmune diseases, including most frequently myasthenia gravis and rarely SLE and other disorders. ${ }^{2}$ Such cases are of considerable interest because of the primary role of the thymus in the immune system. We report here two cases of concurrent SLE and thymoma, which were unusual in that the thymomas were invasive.

\section{Case reports}

CASE 1

A 49-year-old woman was admitted to another hospital in May 1980 with joint and pleuritic chest pains, dyspnoea, and extreme tiredness. One year

Accepted for publication 3 July 1984.

Correspondence to Professor A. Cats, Department of Rheumatology, Academisch Ziekenhuis, Rijnsburgerweg 10, 2333 AA Leiden, The Netherlands. previously she had received antimalarial therapy for similar joint pain, diagnosed as 'seronegative polyarthritis.' and three months previously she had suffered similar but less severe chest pains. Specific inquiry and examination were unremarkable but laboratory tests (Table 1) strongly suggested a diagnosis of SLE. Antibodies against extractable nuclear antigen were absent at this time. In addition detailed investigation including hilar tomography and bronchoscopy were carried out after a chest $x$-ray revealed fluid in the right pleural cavity, non-movement of an elevated left hemidiaphragm, and possible enlargement of the left hilum. No other abnormalities were detected, but the $x$-ray appearances remained unchanged despite a spontaneous improvement in the clinical symptoms. She was treated as an outpatient with non-steroidal antiinflammatory drugs (NSAIDs). Mild symptoms continued without change in laboratory findings and hydroxychloroquine (Plaquenil) $400 \mathrm{mg} /$ daily was recommenced. Pulmonary function tests in April 1981 because of persistent complaints of dyspnoea revealed a restrictive defect (forced vital capacity (FVC) $2420 \mathrm{ml}$ vs. predicted $3140 \mathrm{ml}$ ) with low normal compliance, normal blood gases, and a left basal deficit on ventilation/perfusion scanning consistent with a diaphragmatic palsy. In the absence of 
Table 1 Results of laboratory tests in two patients with SLE and thymoma

Case 1

\begin{tabular}{|c|c|c|c|c|c|c|c|c|c|c|c|}
\hline \multirow[t]{2}{*}{ Test } & \multicolumn{5}{|c|}{ (Months) before thymectomy } & \multirow[t]{2}{*}{ Thymectomy } & \multicolumn{5}{|c|}{ (Months) after thymectomy } \\
\hline & -24 & -19 & -12 & -6 & -2 & & +2 & +6 & +10 & +12 & +18 \\
\hline ESR & 71 & 18 & 26 & 29 & ND & 27 & 87 & 32 & 27 & 37 & 25 \\
\hline Rh factor & - & ND & ND & - & - & - & ND & ND & ND & ND & - \\
\hline LE cells & ++ & ++ & + & ND & ND & ND & ND & ND & ND & ND & ND \\
\hline ANA & \pm & + & + & $1 / 100$ & $1 / 100$ & + & ND & - & + & \pm & ++ \\
\hline Anti-DNA & ++ & + & + & - & - & - & ND & - & - & - & \pm \\
\hline
\end{tabular}

Case 2

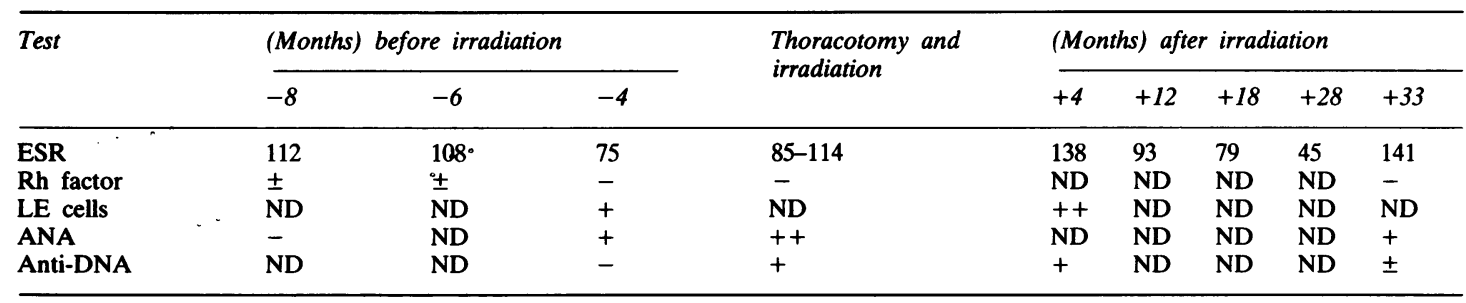

$\mathrm{ND}=$ not done. $-=$ negative or normal. $\pm=$ weak positive. $+=$ moderate positive $++=$ marked positive.

other diagnoses these abnormalities were attributed to the SLE. On further follow-up a trace of proteinuria and microscopic haematuria without casts were detected. After persistent complaints of tiredness and malaise a trial of low-dose corticosteroids (dexamethasone $0.5 \mathrm{mg} /$ day) was instituted, but without effect, and was stopped within a few weeks.

In May 1982 when the patient was admitted to another hospital for gynaecological investigation of abdominal pains a routine chest $x$-ray showed a large central mass. She was transferred for detailed assessment, which showed an anterior mediastinal tumour encroaching on the left hilum and small 'fibrotic' opacities in the left lung field. Bronchoscopy was negative and pulmonary function unchanged apart from slight reduction in gas transfer. At thoracotomy a tumour of the mediastinum invading the left hilum, pericardium, and left lung was discovered. It was removed in toto with the left lung. Pathological examination showed a thymic tumour predominantly composed of epithelial and lymphoid cells with nuclear pleomorphism, prominent nucleoli, and coarsening of the chromatin in the epithelial cells. Invasion of the capsule of an adjacent lymph node was shown histologically, but the resection planes appeared tumour-free. Because of the extensive and invasive nature of the tumour postoperative radiotherapy (50 Gy in five weeks) was given. Serum taken during this admission contained no antiskeletal muscle antibodies. Post- operatively the patient was disproportionately breathless, but pulmonary function tests showed normal arterial oxygen content and the predicted restrictive defect (FVC $1405 \mathrm{ml}$ ). Immunological tests showed no significant changes (Table 1). At outpatient review the patient continued to complain of arthralgia, tiredness, myalgia, and dyspnoea, but laboratory indices were unchanged. Sixteen months after thymectomy increasing dyspnoea was noted, and she was readmitted for assessment. Physical examination revealed little apart from an endinspiratory squeak, but the restrictive ventilatory defect had worsened on testing (FVC $1125 \mathrm{ml}$ ). Detailed radiology revealed no evidence of recurrent tumour, and cardiac catheterisation and pulmonary angiography excluded pulmonary hypertension or emboli. Laryngoscopy revealed a paralysis of the left vocal cord probably residual from the original tumour or surgery. The deterioration was thought to be due to active SLE involving the lung parenchyma, although indices of activity were unchanged. She was treated with methylprednisolone 1 $g$ every second day three times, with dramatic symptomatic and objective improvement (FVC improved to $1750 \mathrm{ml}$ ). She was discharged taking only NSAID's. On two later occasions she developed increased breathlessness and was admitted to hospital. Objective measures were unchanged, but the symptoms improved after further pulse methylprednisolone. 
CASE 2

A 48-year-old woman was referred to hospital in April 1979 for assessment of polyarthralgia and pulmonary abnormalities. Four years previously a routine chest $x$-ray had revealed left hilar enlargement, but tomography had been normal. Two years before referral a symmetrical polyarthritis of the fingers and wrists began and occasional evening fever was noted. Investigations then, apart from a raised ESR, were negative (Table 1 ), and $x$-rays of the joints were normal, but the previously noted left hilar enlargement was still apparent on chest $x$-ray. Over the subsequent two years the antinuclear antibody test (ANA) became strongly positive, and numerous LE cells were seen. No renal abnormalities were detected, and a diagnosis of mild SLE was made. Treatment with NSAIDs was instituted. Follow-up $x$-rays later showed elevation of the left hemidiaphragm, fluid in the pleural space, and parenchymal opacities in the left upper zone, and prompted referral to this hospital for detailed investigation.

Questioning and examination yielded nothing remarkable, but tomography showed a cavitating lesion at the base of the left lung and CT scan parenchymal nodules in the left lung and pleural thickening. Bronchoscopy, mediastinoscopy, sputum cytology, and percutaneous needle biopsy of the lung provided no confirmation of the suspected malignancy, and only chronic inflammatory tissue was seen in the biopsies. Thoracotomy and open lung biopsy were performed, and a mediastinal tumour invading the left lung was found. The tumour was lobular, poorly encapsulated, and composed of a mixture of epithelial cells and lymphocytes. The epithelial cells had vesicular nuclei with moderate pleomorphism, and many mitoses were present. Electron microscopy (Dr D. L. Ruiter) confirmed the diagnosis of thymoma. Examination of the lung showed subpleural metastases with a histological picture similar to that of the thymic tumour, although more of the epithelial cells had a spindle appearance. Because of its apparent slow growth but extensive local invasion high-dose radiotherapy $(50 \mathrm{~Gy})$ of the mediastinum and left lung was considered the optimal therapy. Preradiation pulmonary function was virtually normal (FVC $2830 \mathrm{ml}$ vs. predicted $3050 \mathrm{ml}$ ).

After 20 Gy the patient complained of dyspnoea, right chest pain, arthralgia, and high fever. Examination revealed a pericardial rub. Anaemia was detected ( $\mathrm{Hb} 4.8 \mathrm{mmol} / \mathrm{l}$ : normal 7.5-9.5) and high ESR with a positive Coombs test. Fibrinogen and fibrin deposition products (FDPs) were increased, but no circulating anticoagulant was detected. A chest $x$-ray showed a small right pleural effusion and a marked restrictive ventilatory defect (FVC 1640 $\mathrm{ml}$ ) was detected by pulmonary function testing. Lung scan also showed a right mid zone defect, and because of possible extension of malignancy to the right lung the radiotherapy was stopped. A percutaneous needle biopsy of the suspicious area showed only chronic inflammation, and thoracoscopy failed because of pleural adhesions. In view of the failure to detect malignancy active pulmonary SLE was considered and a trial of corticosteroids begun (prednisone $20 \mathrm{mg} /$ day). There was dramatic clinical improvement, a rise in haemoglobin, and a fall in the ESR, and the right-sided radiological abnormalities disappeared. However, because of the rapid onset of the restrictive ventilatory defects the reserve of pulmonary parenchymal tissue was considered insufficient to allow high-dose radiotherapy to the left lung, and no further irradiation was performed.

The patient was followed up on reducing doses of prednisone, and although she became cushingoid she remained clinically well for 15 months despite a persistently raised ESR, and the chest $x$-ray remained unchanged. Thereafter she developed cough, retrosternal chest pain, and weight loss (13 $\mathrm{kg}$ ) and was readmitted to hospital. Inquiry revealed dysphagia for solids, tiredness, and painful paraesthesiae of the skin. New opacities were seen in the left lung parenchyma on $x$-ray and CT scan, but the vital capacity was well maintained (FVC $2210 \mathrm{ml}$ ). Indices of lupus activity were unchanged, but clotting time and prothrombin time were slightly prolonged. Progression of the thymic tumour was diagnosed, and in view of the limited respiratory reserves chemotherapy was recommended. Over the next six months sequential courses of combination chemotherapy (cyclophosphamide, adriamycin, vincristine, and prednisone) were given with some symptomatic improvement but no radiological change. Thereafter different cytotoxics (procarbazine, mitomycin) were used in place of cyclophosphamide and adriamycin, but fever and pharyngitis developed and she was readmitted to hospital. Treatment with flucloxacillin was begun after a sputum culture grew Staphylococcus aureus, and prednisone was increased $(20 \mathrm{mg} / \mathrm{day})$, but there was clinical deterioration, and new soft opacities appeared on $x$-ray. A bronchoscopy showed candidiasis of the respiratory tract and miconazole was started. In view of possible Pneumocystis carinii infection co-trimoxazole was added, but her condition deteriorated, and she died nearly three years after the original referral.

At necropsy the lungs showed massive infection with Pneumocystis carinii and interstitial pneumonitis combined with necrotising staphylococcal 
pneumonia. The latter had resulted in septic foci in the kidney and heart. The thymic tumour had led to pulmonary metastases, and vital remnants of the tumour were still present in both lungs among much necrosis. The pleural and pericardial cavities were obliterated by adhesions, and there were also peritoneal adhesions to the spleen which were all consistent with a polyserositis due to lupus. No typical lupus abnormalities were found histologically or by immunofluorescence in the skin or kidneys.

\section{Discussion}

Both the cases described had concurrent SLE and invasive thymoma. Although the local incidence of each condition is unknown, the cases were drawn from a population of 53 cases of SLE presenting to a single clinician (H.H.) over a 10 -year period, and during that same decade 21 thymomas were seen in the Department of Pathology. These two cases of concurrent SLE and thymoma appear to represent an increased incidence from that expected by chance, although the association is clearly not common as judged by fewer than 20 case reports in the world literature. ${ }^{3-7}$ Some of the reported cases had other autoimmune diseases in addition. The concurrence, however, is of great theoretical interest in view of the primary immunological role of the thymus and the purported abnormal functioning of $T$ lymphocytes in SLE. ${ }^{1}$ Furthermore the much closer association of myasthenia gravis with thymic tumours ${ }^{2}$ supports the concept of a link between the thymus and autoimmune disease. However, it is not clear in that case whether neoplasia of the thymus is the primary abnormality or whether the heightened glandular activity associated with the immunological disorder predisposes to the formation of tumours. Abnormalities of the thymus have been described in uncomplicated SLE ${ }^{8}$ but thymectomy did not affect the course of the disease. ${ }^{9}$ Neither was surgery or irradiation beneficial to the SLE in the present or in previous cases of concurrent SLE and thymoma, ${ }^{3}$ though a recent report suggested that thymectomy benefited patients with rheumatoid arthritis in whom D-penicillamine treatment had led to the development of myasthenia gravis. ${ }^{10}$ In the latter case thymectomy is frequently beneficial. In addition there was no apparent effect of thymectomy on the serological abnormalities of the SLE, and it is therefore likely that the thymus gland is not the only seat of immunological abnormalities in SLE. But it does seem possible that altered thymic function occasioned by the SLE may lead to tumour formation in a proportion of cases.

Other interesting aspects of these cases concern the difficulty in proving the presence of the suspected mediastinal tumour and in distinguishing relapse of the tumour from active pulmonary SLE. In the first case earlier examination of the mediastinum by øpen operation or mediastinoscopy might have led to an earlier diagnosis. The slow growth of thymic tumours permits 'curative surgery' in most cases diagnosed at an early stage, though both the present cases were unusual in falling into the locally invasive category. ${ }^{2}$ Such staging is best performed by a combination of careful examination during thoracotomy and pathological examination of resection planes to distinguish local tumour invasion from reactive inflammation. Truly malignant tumours with distant metastases appear to be exceptional. Persistent pulmonary symptoms postoperatively may result from either tumour recurrence or pulmonary SLE, and neither pulmonary function tests, radiology nor serology of lupus can be relied on to distinguish the conditions. The possibility of infection in the immunocompromised host must also be considered in the differential diagnosis in some instances. In the absence of overt tumour or infection a trial of steroids with careful monitoring of pulmonary function and serology appears the best course.

The authors thank Dr D. J. Ruiter, Department of Pathology, for carrying out electron microscopy on the surgical specimen from case 2 . Ingrid Veldkamp kindly typed the manuscript.

M. M. Steven is a Boots ARC Travelling Fellow.

\section{References}

1 Fauci A S, Steinberg A D, Haynes B F, Whalen G. Immunoregulatory aberrations in systemic lupus erythematosus. $J$ Immunol 1978; 121: 1473-9.

2 Rosai J, Levine G D. Tumors of the thymus. Atlas of tumor pathology. 2nd series, no 13. Bethesda: Armed Forces Institute of Pathology, 1976: 141-6.

3 Cayla J, Rondier J, Kahan A, Bonvarlet J-P, Fabre P, Le Brigand $\mathrm{H}$. Thymome et maladie lupigue (A propos de deux observations). Rev Rhum Mal Osteoartic 1975; 42: 253-62.

4 Simeone J F, McCloud T, Putman C E, Marsh J. Thymoma and systemic lupus erythematosus. Thorax $1975 ; 30$ : 697-700.

5 Malas D, Weiss S. Progressive multifocal leukoencephalopathy and cryptococcal meningitis with systemic lupus erythematosus and thymoma. Ann Neurol 1977; 1: 188-91.

6 Mastaglia F L, Papadimitriou J M, Dawkins R L, Beveridge B. Vacuolar myopathy associated with chloroquine, lupus erythematosus and thymoma. Report of a case with unusual mitochondrial changes and lipid accumulation in muscle. $J$ Neurol Sci 1977; 34: 315-28.

7 Cooper A, Wells J V. Pemphigus foliaceous, myasthenia gravis and thymoma in a patient with serological evidence of SLE. Aust NZ J Med 1981; 11: 277-80.

8 Goldstein G, Mackay I R. The thymus in systemic lupus erythematosus; a quantitative histopathological analysis and comparison with stress evolution. $\mathrm{Br}$ Med J 1967; ii: 475-8.

9 Mackay I R, Goldstein G, MacConchie I H. Thymectomy in systemic lupus erythematosus $\mathrm{Br}$ Med $J$ 1963; ii: 792-4.

10 Szobor A. Benefit of thymectomy in immune diseases other than myasthenia. Lancet 1984; i: 277-8. 